\title{
OR-19
}

\section{Effect of Moisture Content on Microbial Contamination in Orthosiphon Stamineus (BENTH) Leaf Water Extract Powder}

\author{
Mahibub M. Kanakal 1,2,3, Amin MS Abdul Majid ${ }^{2}$, Aman S Abdul Majid ${ }^{3,{ }^{*}}$, Munavvar Abdul Sattar ${ }^{2}$ \\ and Noor Shahida Ajmi ${ }^{4}$ \\ ${ }^{I}$ Department of Pharmaceutics, Faculty of Pharmacy, Universiti Teknologi MARA, Bertam Campus, Kepala Batas, \\ 13200 P.Pinang, Malaysia; ${ }^{2}$ School of Pharmaceutical Sciences, Universiti Sains Malaysia, 13200 P.Pinang, Malaysia; \\ ${ }^{3}$ Cluster of Integrative Medicine, Advanced Medical and Dental Institute, Universiti Sains Malaysia, Kepala Batas, \\ 13200 P.Pinang, Malaysia; ${ }^{4}$ Department of Pharmacy, Allianze University College of Medical Sciences (AUCMS), \\ 13200 Kepala Batas, P. Pinang, Malaysia; E-mail: amanshah75@yahoo.com
}

Water based plant extracts are widely used for various medicinal applications. Due to its simple and economical extraction process, herbal based industries prefer water based extraction. The water based extracts are prone to microbial growth due to presence of moisture as they are hygroscopic in nature. Microbial growth in herbal extracts can be controlled using suitable excipients. This study investigates and compares microbial contamination tendency in formulated and unformulated herbal preparations with respect to their moisture content. Water based leaf extract powder of Orthosiphon Stamineus (Benth) was selected. Moisture content and microbial limit tests were performed immediately for the fresh extract powder and in its final formulation. Both the extract and formulation were stored at room temperature for three months exposed and unexposed to air. Microbial population and weight changes were monitored on a dynamic moisture balance over a period of 3 months. The study revealed that the unexposed formulation still contained moisture however had less moisture content and microbial growth. The findings provide an insight into a possible approach for formulating moisture-sensitive pharmaceutical products, especially dry powder preparations for use in tropical countries with high humidity environment.

Keywords: Moisture content, Microbial contamination, Water extract. 